\title{
Proposal for Generalized Exergy and Entropy Properties Based on Stable Equilibrium of Composite System-Reservoir
}

\author{
Pierfrancesco Palazzo \\ Technip, Rome, Italy \\ Email:ppalazzo@technip.com
}

Received April 20, 2013; revised May 25, 2013; accepted June 27, 2013

Copyright (C) 2013 Pierfrancesco Palazzo. This is an open access article distributed under the Creative Commons Attribution License, which permits unrestricted use, distribution, and reproduction in any medium, provided the original work is properly cited.

\begin{abstract}
The present theoretical study represents a proposal aimed at investigating about the possibility of generalizing the canonical entropy-exergy relationship and the reservoir concept. The method adopted assumes the equality of pressure and chemical potential as necessary conditions of mutual stable equilibrium between a system and a reservoir in addition to the equality of temperature that constitutes the basis for defining entropy as deriving from energy and exergy concepts. An attempt is made to define mechanical and chemical entropy as an additional and additive component of generalized entropy formulated from generalized exergy property. The implications in exergy method and the possible engineering applications of this approach are outlined as future developments among the domains involved.
\end{abstract}

Keywords: Adiabatic Availability; Available Energy; Generalized Exergy; Generalized Entropy; Reservoir; Stable Equilibrium; Exergy Method

\section{Introduction}

The formulation of the Second Law is based on the existence and uniqueness of the state of stable equilibrium of a system interacting with a reservoir "among all the states of a system that have a given value of the energy and are compatible with a given set of values of the amounts of constituents and of the parameters" [1]. This general statement requires compliance with the necessary conditions of equality of (absolute) temperature, pressure and chemical potential between system and reservoir. The Second Law implies the definition of entropy property in relation to temperature. The entropy of system $A$ can also be derived as the difference of energy $E$ and exergy $E X$ associated with the generalized available energy $\Omega^{R}$ with respect to a reservoir $R$ at constant temperature $T_{R}$ [1]. The Second Law, expressed as indicated above, has to account for pressure and chemical potential in addition to temperature, hence the purpose of this present study is to conceive a generalized formulation of exergy, and consequently of entropy, based on equality of pressure and chemical potential, in addition to temperature, between a system $A$ and a reservoir $R$ at constant temperature $T_{R}$, constant pressure $P_{R}$ and constant chemical potential $\mu_{R}$.

\section{Theoretical Model and Method}

The following assumptions are posited: 1) the analysis is focused on "simple systems" according to the terminalogy and definitions adopted by Gyftopoulos and Beretta [1]; 2) the external reference system behaves as a reservoir as defined in the literature [1]; 3) the system may be large or small, even at molecular level, and may experience states of equilibrium and nonequilibrium [1]; 4) physical and chemical exergy are accounted for; 5) kinetic energy and the potential energy of the system as a whole are neglected. These assumptions are posited in order to constitute the requirements complying with the fundamental framework to which the addressed literature refers.

The method adopted assumes the equality of pressure and chemical potential of the composite system-reservoir $A R$ as additional necessary conditions for mutual stable equilibrium, other than equality of temperature. The formulation of the entropy property is derived from exergy, related to a reservoir at constant temperature, constant pressure and constant chemical potential. This set of generalized potentials equality constitutes a necessary and sufficient condition for the stable equilibrium of a composite system-reservoir consistent with the Second Law 
statement.

\section{Thermal Entropy Derived from Thermal Exergy}

The behaviour of the exergy property is characterized by additivity because it is defined considering an external reference system or an internal part of the system itself that behaves as a reservoir [1,2]. Exergy is derived from the generalized available energy that is a consequence of the concept of adiabatic availability when the system interacts with a reservoir [1]; the definitions of available energy and exergy are based on the mutual stable equilibrium of a system $A$ with a reservoir $R$ at constant temperature $T_{R}$ and account for work interaction by means of a weight process; this implies that, as defined in these terms, (thermal) exergy expresses the maximum net useful work $\left(W_{10}^{A R \rightarrow}\right)^{\mathrm{MAX}}$ connecting two states 0 and 1 obtained by means of a weight process resulting from the available energy between variable temperatures $T$ of system $A$ and constant temperature $T_{R}$ of reservoir $R$ as expressed by the following equations adopting the symbology used by Gyftopoulos and Beretta [1]:

$$
\begin{aligned}
& E X^{T}=\left(W_{10}^{A R \rightarrow}\right)^{\mathrm{MAX}}=\left(\Omega_{1}^{R}-\Omega_{0}^{R}\right)^{\mathrm{MAX}} \\
& =\left(U_{1}-U_{0}\right)-T_{R} \cdot\left(S_{1}^{T}-S_{0}^{T}\right)+P_{R} \cdot\left(V_{1}-V_{0}\right)
\end{aligned}
$$

The definition of entropy is derived from the difference between energy and available energy [1] and is here defined as thermal entropy $S^{T}$ by virtue of the considerations discussed above:

$$
S_{1}^{T}-S_{0}^{T}=\frac{1}{T_{R}}\left[\left(E_{1}-E_{0}\right)-\left(\Omega_{1}^{R}-\Omega_{0}^{R}\right)\right]^{T}
$$

These equations, reported in the addressed literature, constitute the outset of the present study underpinned by the consideration that equality of temperature is a necessary condition that is not sufficient to prove that a system is in stable equilibrium with the reservoir. Indeed, even though two interacting systems are in thermal stable equilibrium owing to equality of temperatures, the two systems may experience nonequilibrium states due to the non-null difference between pressures or chemical potentials. The equality of total potential and pressure between system and reservoir should therefore constitute the set of additional necessary conditions to ensure the stable equilibrium so that the equality of the complete set of "generalized potentials" constitutes a necessary and sufficient condition for mutual stable equilibrium in compliance with the Second Law as worded by Gyftopoulos and Beretta [1]. As a consequence of the procedure adopted for its definition by the said Authors [1], entropy is an additive property that can be generalized to include the contribution of additional components fulfilling to the conditions of equality of pressure and chemical potential that guarantee the mutual stable equilibrium of the composite system-reservoir.

An intuitive rationale of the procedure here adopted to define entropy may be explained considering that internal energy is characterized by a "hybridization" of ordered and disordered (due to distribution of molecule' position and velocity of system's particles) energy status. Entropy may be regarded as the measure of the amount of disordered energy - released to the reservoir - resulting from the difference of hybrid energy - ordered and disordered - and available energy - ordered energy - transferred, as useful interaction, to the external system.

\section{Mechanical Entropy Derived from Mechanical Exergy}

The formulation of thermal exergy in Equation (1) expresses the weight process as the result (and the measure) of the maximum net useful work that can be extracted from a system interacting with a thermal reservoir. The weight process can also be regarded as the minimum net useful work delivered to the system and hence can be associated with the maximum net useful heat according to the concepts of equivalence and interconvertibility [3-5]. In this case, the weight process is calculated as the result of the interaction of the system with a mechanical reservoir at constant pressure $P_{R}$. The mechanical reservoir is here assumed to possess the same properties as a thermal reservoir $[1,2,6]$ that is characterized and defined without explicit reference to any specific form of generalized potential.

The equality of pressure between system and reservoir is assumed to be an additional necessary condition of mutual stable equilibrium. This condition should therefore be complied with equality of temperature to ensure that the status of composite system-reservoir is one of stable equilibrium.

If the concept of generalized available energy $\Omega^{R}$ [1] is now referred to, then the formulation of mechanical exergy should be translated into the following expression where the superscript " $M$ " stands for "Mechanical reservoir" since the composite system-reservoir undergoes a "work interaction" and the physical meaning becomes the "maximum" net useful heat of the system:

$$
E X^{M}=\left(Q_{10}^{A R \rightarrow}\right)^{\mathrm{MAX}}=\left(\Omega_{1}^{R}-\Omega_{0}^{R}\right)^{M}
$$

The definition of mechanical exergy is the basis for deriving the expression of mechanical entropy using the procedure adopted for thermal exergy and thermal entropy:

$$
S_{1}^{M}-S_{0}^{M}=\frac{\bar{R}}{P_{R} V_{R}}\left[\left(E_{1}-E_{0}\right)-\left(\Omega_{1}^{R}-\Omega_{0}^{R}\right)\right]^{M}
$$


This expression of mechanical entropy can be demonstrated considering that the procedure conceived by Gyftopoulos and Beretta proving the formulation of (thermal) entropy [1], does not impose any restriction with respect to the form of energy and generalized available energy that constitute the expression of entropy. Therefore the same procedure can be considered valid regardless of the physical nature of the properties involved. The minimum amount of weight process corresponds to the maximum amount of heat interaction:

$$
\begin{aligned}
& {\left[\left(E_{1}-E_{0}\right)-\left(\Omega_{1}^{R}-\Omega_{0}^{R}\right)\right]^{M}} \\
& =\left(W_{10}^{A R \leftarrow}\right)^{\mathrm{MIN}}=P_{R} V_{R}\left(\ln V_{1}-\ln V_{0}\right)
\end{aligned}
$$

which expresses the (minimum) amount of work interaction absorbed from mechanical reservoir at constant pressure $P_{R}$ and constant $V_{R}$ (total volume coincides with specific volume in the particular case of a reservoir). Equation (5), substituted in the former relation, expresses the mechanical entropy:

$$
S_{1}^{M}-S_{0}^{M}=\bar{R}\left(\ln V_{1}-\ln V_{0}\right)
$$

\section{Chemical Entropy Derived from Chemical Exergy}

The chemical potential generated by interaction among the molecules of an open system constitutes a component of internal energy and is defined as a form of "disordered energy", as thermal energy is [7].

The maximum work of a reversible (internally and externally) chemical reaction is expressed by the Gibbs function so that $W_{R E V}^{N E T}=-\Delta G$. The Van't Hoff equilibrium open system at constant temperature and constant pressure is a suitable device for reproducing a typical chemical reaction where $A, B$ are reactants and $C, D$ are products [7]:

$$
n_{A} A+n_{B} B \leftrightarrow n_{C} C+n_{D} D \Rightarrow \ln K_{P}=-\frac{\Delta G_{0}}{\bar{R} T_{0}}
$$

is adopted to express maximum work taking into account the internal mechanism of chemical reaction by means of the expression depending on the equilibrium constant $K_{P}$ of the reaction.

The Gibbs relation, obtained from mass and internal energy balance, is as follows:

$$
\begin{aligned}
& \mathrm{d} U=T \cdot \mathrm{d} S-P \cdot \mathrm{d} V+\sum_{i} \mu_{i} \mathrm{~d} n_{i} \\
& =\left(\frac{\partial U}{\partial S}\right)_{V, n} \mathrm{~d} S-\left(\frac{\partial U}{\partial V}\right)_{S, n} \mathrm{~d} V+\sum_{i}\left(\frac{\partial U}{\partial n_{i}}\right)_{V, S, n} \mathrm{~d} n_{i}
\end{aligned}
$$

where $\mu_{i}=\left(\frac{\partial U}{\partial n}\right)_{V, S, n}$ represents the chemical potential of the i-th constituent.

Chemical exergy is defined by Kotas [7] as "the maximum work obtainable from a substance when it is brought from the environmental state to the dead state by means of processes involving interaction only with the environment". The chemical reservoir can be characterized according to the definition proposed by Gyftopoulos and Beretta [1] as a "reservoir with variable amounts of constituents". The whole chemical and physical process is modelled by a schema subdivided in two typical steps [7]: the initial molecular system form's rearrangement (molecular structure) and the final molecular system's dimensional change (geometry-kinematics or pressure and temperature). The two representative processes are: chemical reaction open system and physical operation open system; the two processes in series provide an expression of maximum net useful work withdrawn from the system expressed by Equation [7]:

$$
E X^{C}=\left(W_{10}^{A R \rightarrow}\right)^{\mathrm{MAX}}=\bar{R} T_{R} \ln \frac{P_{1}}{P_{0}}
$$

Mass interaction is the characteristics of the chemical energy transfer and is moved by the difference of chemical potential between the system and the reservoir.

In the more general case of a mixture of $n$ chemical constituents:

$$
E X^{C}=\sum_{i}^{n}\left(W_{10}^{A R \rightarrow}\right)_{i}^{\mathrm{MAX}}=\bar{R} T_{R} \sum_{i}^{n} x_{i} \ln x_{i}
$$

The equality of total potentials is accounted for as an additional necessary condition of mutual stable equilibrium between the system and the reservoir other than the equality of temperature [1]. This implies a definition of chemical entropy derived from chemical exergy and chemical energy in line with the methodology previously adopted.

If the concept of generalized available energy is now again considered, the formulation of chemical exergy should be translated into the following expression:

$$
E X^{C}=\left(W_{10}^{A R \rightarrow}\right)^{\mathrm{MAX}}=\left(\Omega_{1}^{R}-\Omega_{0}^{R}\right)^{C}
$$

where the superscript " $C$ " stands for "Chemical reservoir" since the composite of system and reservoir undergoes a "mass interaction".

Now that chemical exergy is defined, and considering that entropy is an additive property, the expression used for entropy associated with heat interaction can be extended to chemical potential depending on mass interaction:

$$
\left(S_{1}-S_{0}\right)^{C}=\frac{1}{\mu_{R}}\left[\left(E_{1}-E_{0}\right)-\left(\Omega_{1}^{R}-\Omega_{0}^{R}\right)\right]^{C}
$$

This constitutes the expression of the chemical entropy derived from chemical exergy based on the equality of 
chemical potential which constitutes a necessary condition for mutual stable equilibrium between the system and the chemical reservoir.

Since entropy is an inherent property of all systems [8-11], chemical entropy would be characterized by the chemical potential of all atoms and sub-molecules that constitute all compounds and determine the supra-molecular architecture and configurations of all molecular systems. The dimensions and shapes of molecular structures play, in this perspective, a fundamental role in determining the minimum entropy level that ensures the stability of matter and its capability to react with other co-reactants, as well as to undergo endogenous or exogenous processes. Typical is organic chemistry and biochemistry in which same chemical formula are incapable of describing materials and compounds that display different physical and chemical characteristics and properties. The vibration degree of freedom is an additional aspect correlated to molecular structure complexity.

\section{Generalized Exergy and Entropy}

The definition of a thermo-chemical-mechanical reservoir characterized by constant temperature, pressure and chemical potential implies the additivity $[1,2]$ of the components constituting the generalized exergy:

$$
E X^{G}=E X^{T}+E X^{M}+E X^{C}
$$

The internal energy balance of the composite systemreservoir, adopting the symbology in [1], provides the amount of weight process due to thermal, mechanical and chemical contributions:

$$
\begin{aligned}
E X^{G}= & -\left(W^{A R \rightarrow}\right)-\left(Q^{A R \rightarrow}\right)-\left(M^{A R \rightarrow}\right) \\
= & \Delta U^{\text {SYSTEM }}+\Delta U^{\text {RESERVOIR }} \\
= & \Delta U_{Q}^{\text {SYSTEM }}+\Delta U^{R, Q}+\Delta U_{W}^{\text {SYSTEM }}+\Delta U^{R, W} \\
& +\Delta U_{C}^{\text {SYSTEM }}+\Delta U^{R, C}
\end{aligned}
$$

where: $\Delta U^{R, Q}=Q_{R}$ is the minimum heat interaction with the thermal reservoir; $\Delta U^{R, C}=M_{R}$ is the minimum mass interaction with the chemical reservoir and $\Delta U^{R, W}=W_{R}$ is the minimum work interaction with the mechanical reservoir.

The isothermal process realizes energy conversion and at the same time an entropy conversion from thermal entropy to mechanical entropy that occurs due to simultaneous heat interaction and work interaction. Both conversions are accounted for in the physical exergy expression:

$$
\begin{aligned}
E X^{G}= & \Delta U^{\text {SYSTEM }}+\Delta U^{\text {RESERVOIR }} \\
= & \left(U-U_{0}\right)-T_{R} \cdot \Delta S^{\text {TOTAL }, R}+P_{R} \cdot \Delta V^{R} \\
& -T_{R} \cdot \Delta S^{T, R}+\mu_{R} \cdot \Delta S^{C, R}
\end{aligned}
$$

where the term $-T_{R} \cdot \Delta S^{\mathrm{TOTAL}, R}$ represents the contribution to entropy conversion only occurring inside the reservoir and the terms $P_{R} \cdot \Delta V^{R}-T_{R} \cdot \Delta S^{T, R}+\mu_{R} \cdot \Delta S^{C, R}$ represent the contribution transferred from the system to the reservoir. It is noteworthy that entropy conversion is inherent in energy conversion and that entropy conversion requires the additional term that contributes to exergy balance expressed in the above formulation which therefore considers the effect of both energy and entropy conversion processes. Considering that entropy is an additive property constituted by components deduced from the corresponding generalized exergy's components, the generalized entropy may be defined as the sum of thermal, mechanical and chemical terms: $S^{G}=S^{T}+S^{M}+S^{C}$.

\section{Remarks upon the Gibbs-Duhem Relation}

The Gibbs-Duhem relation [1] constitutes a condition among all intensive properties temperature-pressure and chemical potential-that define the state of a heterogeneous system. If the system is homogeneous and constituted by one constituent only, there are no phase changes or chemical reaction mechanisms inside the system implying the system itself is at chemical equilibrium and the Gibbs-Duhem relation is:

$$
S \mathrm{~d} T-V \mathrm{~d} P+n \mathrm{~d} \mu=0
$$

Chemical potential $\mu$ is defined as the component of internal energy generated by the interactions of inter-particle positions and relative distance, except for kinetic potential (due to inter-particle relative velocity). Considering these assumptions, the system model characteristics can be assimilated to those adopted in the Kinetic Theory of Gas [12] which, in particular, considers molecules undergoing elastic repulsive interaction forces (LennardJones) on collision with other molecules and with the wall of the container but otherwise exert no attractive interaction forces (Van der Waals) on each other or on the container wall [13]. The container walls represent a geometrical volume constraint condition imposed on the system.

In the special case of the system's undergoing an isothermal process, the pressure changes are due to the change of volume that determines the frequency of particle collisions [12]; if the system is characterized as assumed, then the differential of chemical potential among all atoms or molecules is due to the temperature that is the only inter-particle kinetic energy transformed into inter-particle potential energy due to repulsive collision interactions (attractive interactions are negligible by assumption). Considering that no chemical reactions occur inside the system as assumed, then $\mathrm{d} \mu=0$, as reported by Kotas [6] for systems with a fixed chemical composi- 
tion, and the Gibbs-Duhem relations becomes:

$$
S \mathrm{~d} T=V \mathrm{~d} P
$$

where $S$ and $V$ are not null both being inherent properties of any system [8]. If the system undergoes an isothermal reversible process, then the temperature remains constant by definition, so that $\mathrm{d} T=0$; on the other side, $\mathrm{d} P$ is not null in the same isothermal process and therefore an inconsistency appears. The same inconsistency is displayed if an isobaric process is accounted for using the Gibbs-Duhem relation where pressure remains constant, so that $\mathrm{d} P=0$, and temperature does not. This inconsistency involves the intensive properties temperature and pressure which determine the thermo-mechanical conversions involved in the concept of available energy and exergy. The alternative approach set forth in the present study resolves this inconsistency.

The chemical potential expressed by the Gibbs function reveals that entropy property is variable in the isothermal process and consequently the chemical potential is not constant, which is in contradiction with the assumption set forth.

The chemical potential that appears in assumed system model is due exclusively to the repulsive interactions on collisions and depends solely on molecules' velocity, so that temperature constitutes the first contribution to pressure. The second contribution is due to the (specific) volume determining the frequency of collisions between molecules and the external system which, on the other side, does not determine the chemical potential due to attractive interactions among the molecules which does not exist in the model assumed.

If $\mathrm{d} \mu=0$ and $\mathrm{d} T=0$ and considering attractive interactions as negligible, repulsive interaction potential is equal to kinetic potential (transformation during collision only). The Gibbs relation:

$$
\mathrm{d} U=T \cdot \mathrm{d} S-P \cdot \mathrm{d} V=\delta Q+\delta W
$$

can be reformulated in different terms adopting thermal entropy and mechanical entropy as defined in Equation (6):

$$
\mathrm{d} U=T \cdot\left(\mathrm{d} S^{T}\right)-\frac{P V}{\bar{R}} \cdot\left(\mathrm{d} S^{M}\right)=\delta Q+\delta W
$$

and transformed, by using the state equation $P V=\bar{R} T$ valid in this special case, and consistent with the Kinetic Theory of Gases [12], in the following form:

$$
\begin{aligned}
& \mathrm{d} U=T \cdot\left(\mathrm{d} S^{T}\right)-T \cdot\left(\mathrm{d} S^{M}\right) \\
& =T \cdot\left(\mathrm{d} S^{T}-\mathrm{d} S^{M}\right)=\delta Q+\delta W
\end{aligned}
$$

This, associated with the temperature, takes into account either heat or work interactions contributing to variations in internal energy.
It is noteworthy that, in the case of work interaction, the work put into the system, which is considered positive, corresponds to a decrease of mechanical entropy as per Equation (6). This is the opposite of heat interaction that is positive if thermal entropy increases. In other terms, heat input causes thermal entropy to increase, work input causes mechanical entropy to decrease, therefore work depends on pressure in the opposite manner that heat depends on temperature. If the system releases work, it increases mechanical entropy because mechanical energy, distributed among the particles that constitute the system, progressively becomes similar to thermal energy i.e. energy distributed by the velocity of the same particles. Increasing volume means that pressure is progressively determined by particles' kinetic energy (temperature) with respect to the contribution of the frequency of collisions among particles and with the external system surface determined by the volume. Pressure is thus progressively the more like temperature as volume increases.

The balance of entropy, along the isothermal expansion reversible process $\mathrm{d} U=0$, is deduced from the equation $S^{\text {TOTAL }}=S^{T}-S^{M}=0$ where $S^{T}$ is the thermal entropy input due to heat flowing into the system and $-S^{M}$ is the mechanical entropy output from the system which is the opposite of the mechanical entropy input flow inherent in the expansion work output from the system itself. The physical meaning is that conversion from a kinetic form into a geometric form, required to convert heat into work, necessitates an increase in mechanical entropy.

Due to the fact that mechanical entropy must enter the system because of work output, then the mechanical entropy direction is inverted compared to the thermal entropy direction in the system's entropy balance. Total entropy is consequently constant if either thermal and mechanical entropy enter into the system. The rationale of this statement is that these two entropy components have an opposite origin and elide each other.

If the term $\Delta S^{\text {TOTAL }}$ is expressed by means of $S^{\text {TOTAL }}=S^{T}-S^{M}=0$, the total entropy, resulting from the addition of thermal and mechanical components of entropy, implies that thermal entropy would be constant in an isothermal reversible process that requires heat interaction by means of thermal entropy exchange.

On the other side, pressure does not derive from inter-particle chemical potential and is just the mechanical effect produced by the temperature itself (apparent potential).

The Gibbs relation, expressed in terms of the Equation (19), resolves the apparent inconsistency highlighted in the Gibbs-Duhem relation. In fact, this can be reformulated as follows: 


$$
\begin{aligned}
& \mathrm{d} U=T\left(\mathrm{~d} S^{T}-d S^{M}\right)+\mu \cdot \mathrm{d} n \\
& =T \mathrm{~d} S^{T}-T \mathrm{~d} S^{M}+\mu \cdot \mathrm{d} n
\end{aligned}
$$

The Euler relation is obtained from the Gibbs relation by integration at constant temperature and constant chemical potential [1], so that:

$$
\begin{aligned}
& U=T S^{\text {TOTAL }} \\
& =T \cdot\left(S^{T}-S^{M}\right)+\mu \cdot n=T S^{T}-T S^{M}+\mu \cdot n
\end{aligned}
$$

If compared with the classical expression of the Gibbs relation $U=T S+\mu \cdot n-P V$, the term $-P V$ corresponds to the term $-T S^{M}$. Moreover, in the case of an isothermal process (and absence of chemical reactions so that chemical potential is constant) it requires that total entropy is constant also implying that thermal entropy variation is equal to mechanical entropy variation.

It is noteworthy that, in the case of an ideal system as assumed, internal energy $U$ is associated with the kinetic energy of the molecules, and thus to temperature only; however, internal energy components depend on the terms $\mu \cdot n$ and $-P V$ which both depend on volume as well. This dependence ensures that real systems in which thermodynamic conditions are affected by interactions among molecules that determine the potential energy, are characterized by volume. In differential terms, the Euler relations are:

$$
\begin{aligned}
& \mathrm{d} U \\
& =T \mathrm{~d} S^{\text {TOTAL }}+S^{\text {TOTAL }} \mathrm{d} T+\mu \cdot \mathrm{d} n+n \cdot \mathrm{d} \mu \\
& =T \cdot\left(\mathrm{d} S^{T}-\mathrm{d} S^{M}\right)-\left(S^{T}-S^{M}\right) \cdot \mathrm{d} T+\mu \cdot \mathrm{d} n+n \cdot \mathrm{d} \mu \\
& =T \mathrm{~d} S^{T}-T \mathrm{~d} S^{M}+S^{T} \mathrm{~d} T-S^{M} \mathrm{~d} T+\mu \cdot \mathrm{d} n+n \cdot \mathrm{d} \mu
\end{aligned}
$$

On combining the Gibbs relation and Euler relation expressed in the terms set forth, the Gibbs-Duhem relation $S \mathrm{~d} T-V \mathrm{~d} P+\mu \cdot \mathrm{d} n=0$ assumes the form:

$$
\begin{aligned}
& S^{T} \mathrm{~d} T-S^{M} \mathrm{~d} T+n \mathrm{~d} \mu \\
& =\left(S^{T}-S^{M}\right) \cdot \mathrm{d} T+n \cdot \mathrm{d} \mu \\
& =S^{\text {TOTAL }} \cdot \mathrm{d} T+n \cdot \mathrm{d} \mu=0
\end{aligned}
$$

where: $T$ constitutes the inter-particle kinetic potential component of internal energy resulting in the $P V$ macroscopic work interaction transferred by means of a weight process; $\mu$ constitutes the inter-particle chemical potential component of internal energy resulting in the $P V$ macroscopic work interaction transferred by means of a weight process. The kinetic and chemical constitute the two fundamental potentials at microscopic inter-particle level interacting at macroscopic level that constitute the hierarchical geometric and kinematic structure.

The dualism of kinetic potential and chemical potential constitutes the inherent structure of potentials even in the special case of an ideal system for which inter-particle potential energy in null. In this case, in fact, potential energy still exist in the form of repulsive reaction potential energy that is due to kinetic energy transformed on collision only, without macroscopic effects on the entire system.

This different form of the Gibbs-Duhem relation resolves the apparent inconsistency in the special case of the isothermal ideal process. In fact, $n \cdot \mathrm{d} \mu=0$ because the system model is ideal and $\mathrm{d} T=0$ remains the only condition to be satisfied since $\mathrm{d} P$ no longer appears in the Gibbs-Duhem relation as expressed in Equation (23). The rationale of these statements can also be found in the behaviour of elements, molecules and atoms, constituting the system as a whole. In fact, in the isothermal process, the temperature and subsequent intermolecular repulsive interactions on each collision are constant and the variations of kinetic potential and chemical potential (due to intermolecular repulsive interactions) are therefore null: $\mathrm{d} T=0$ and consequently $\mathrm{d} \mu=0$. In the case of the isobaric process, temperature and pressure are variable or kinetic potential and chemical potential (due to repulsive interactions at each inter-particle collision) both change along the isobaric process: $\mathrm{d} T \neq 0$ and consequently $\mathrm{d} \mu \neq 0$. Even in the case of an ideal system, there is dualism and symmetry of kinetic energy and potential energy among the molecules so that $\mathrm{d} T>0$ and $\mathrm{d} \mu>0$.

Pressure is the mechanical effect of the contribution related to kinetic interaction and related potential and chemical interaction and related potential. In this perspective, pressure can viewed as the outcome of the temperature and chemical potential of a complex multi-particle system, converted into work interaction with the external reservoir and with the external weight process.

Finally, notwithstanding the restrictions assumed for the model adopted, the behaviour of the system is coherent with expectations in terms of phenomena and tendency of the properties in the general case of real systems and processes where each particle experiences attractive interaction with all others, and does not contradict the fundamentals reported in the literature.

\section{Conclusion}

The three additive components of exergy discussed in this study constitute the components of generalized exergy that depends on temperature, pressure and chemical potential and, at microscopic level, on the kinetic energy and potential energy generated by interactions among the molecules of the system. Moreover, three components of entropy property have been inferred from the corresponding exergy components. In particular, chemical exergy and entropy are correlated to the molecular structure of matter due to the composite of molecules geome- 
try and chemical bonds characteristics. The aim of seeking a property related to molecular or supra-molecular architecture is to obtain a method able to predict a-priori stability as well as capability in self-assembling processes and the related intermediate phases of chemical compounds that are not available in the environment and which could undergo a building process by means of nano-sciences technologies. Such a method would make it possible to design materials characterized by properties that could be evaluated prior to being realized and to confirm predictions by means of experiments and laboratory tests. Finally, the method of Entropy Generation Minimization (EGM) [14-16] associated with the Extended Exergy Accounting (EEA) $[17,18]$ could be further generalized to provide an overarching paradigm for analysing the whole process.

\section{REFERENCES}

[1] E. Gyftopoulos and G. P. Beretta, "Thermodynamics: Foundations and Applications," Dover Publications, New York, 2005.

[2] R. A. Gaggioli, International Journal of Applied Thermodynamics, Vol. 1, 1998, pp. 1-8.

[3] W. R. Dunbar, N. Lior and R. A. Gaggioli, Journal of Energy Resources Technology, Vol. 114, 1992.

[4] R. A. Gaggioli, D. H. Richardson and A. J. Bowman, Journal of Energy Resources Technology, Vol. 124, 2002, pp. 105-109. doi:10.1115/1.1448336

[5] R. A. Gaggioli and D. M. Paulus Jr. “Available EnergyPart II: Gibbs Extended,” Transaction of the ASME 2002,
2002.

[6] R. A. Gaggioli, “The Dead State,” Proceedings of 25th ECOS, Perugia, 2012.

[7] T. J. Kotas, "The Exergy Method of Thermal Plant Analysis,” Reprint Edition, Krieger Publishing Company, Krieger, 1995.

[8] E. P. Gyftopoulos, International Journal of Thermodynamics, Vol. 9, 2006, pp. 107-115.

[9] G. P. Beretta, International Journal of Thermodynamics, 2008.

[10] E. Zanchini, International Journal of Thermodynamics, 2010.

[11] E. Zanchini and G. P. Beretta, International Journal of Thermodynamics, Vol. 13, 2010, pp. 67-76.

[12] H. A. Jakobsen, “Chemical Reactor Modeling," SpringerVerlag, Berlin, Heidelberg, 2008. doi:10.1007/978-3-540-68622-4_2

[13] B. H. Bransden and C. J. Joachain, "Physics of Atoms and Molecules,” 2nd Edition, Prentice Hall, Upper Saddle River, 2003.

[14] T. L. Hill, "Statistical Mechanics. Principles and Selected Applications,” Dover, New York, 1987.

[15] E. Sciubba, Entropy, Vol. 12, 2010, pp. 1885-1866. doi:10.3390/e12081855

[16] E. Sciubba, International Journal of Thermodynamics, Vol. 14, 2011, pp. 11-20.

[17] M. Belli and E. Sciubba, International Journal of Exergy, Vol. 5, 2006.

[18] E. Sciubba, Energy, Vol. 28, 2003, pp. 1315-1334. doi:10.1016/S0360-5442(03)00111-7 